\title{
Synthesis, Characterization, and Antibacterial Activity of Some Novel 5-Chloroisatin Derivatives
}

\author{
Z. Tribak ${ }^{1}$, O. El Amin ${ }^{2}$, M.K. Skalli ${ }^{1 *}$, O. Senhaji ${ }^{3}$, Y. Kandri. Rodi ${ }^{4}$, \\ M. Houssaini. Iraqui ${ }^{2}$ \\ ${ }^{1}$ Laboratory of Applied Chemistry, Sidi Mohamed Ben Abdellah University, Faculty of Sciences and Technology \\ of Fes, Morocco \\ ${ }^{2}$ Laboratory of Microbial Biotechnology, Faculty of Science and Technology, B.P. 2202, Fes, Morocco \\ ${ }^{3}$ Laboratory of Applied Physical Chemistry, Moulay Ismaïl University, Faculty of Sciences and Technology of \\ Errachidia, Morocco. \\ ${ }^{4}$ Laboratory of Applied Organic Chemistry, Sidi Mohamed Ben Abdellah University, Faculty of Science and \\ Technology of Fes, Morocco
}

ABSTRACT: The development of potential antibacterial requires the synthesis of a new series of 5Chloroisatin derivatives incorporating various aromatic aldehydes in the case 1,3-Dipolar Cycloaddition including Nitrile oxide, as well as the cycloaddition Alcyne-Azide Catalytic with Copper. The charcterization of the structure of the synthesized compounds was confirmed by means of their IR, ${ }^{1} \mathrm{H}-\mathrm{NMR}$ and ${ }^{13} \mathrm{C}-\mathrm{NMR}$ spectral data. In addition, the antibacterial properties in vitro were tested against certain microorganisms using the disk diffusion technique. A majority of compounds show better activity against several of the microorganisms.

Keywords: 5-Chloroisatin derivatives, 1,3-Dipolar Cycloaddition, synthesis, antibacterial properties.

\section{INTRODUCTION}

The use of antibiotics is a common phenomenon throughout the world, due to the growth of microbial infections where these diseases are a frequent cause of death in contemporary medicine [1]. This requires exploring and synthesizing a new class of effective antimicrobial compounds that are part of the family of 5-Chloroisatin derivatives against pathogenic microorganisms that have developed resistance to antibiotics used in the current regime. The 5-Chloroisatin derivates are an important class of organic compounds, some of which show significant biological like antibacterial, antiviral, antifungal [2], anti-inflammatory [3], analgesic [4], anti-tubercular [5] and antidepressant [6]. In view of these facts, we envisaged synthesizing new dipolarophiles, isoxazoles and 1, 2, 3-triazoles derived from 5Chloroisatin, successively by the following methods, $\mathrm{N}$-alkylation, 1, 3-Dipolar Cycloaddition by nitrile oxides and Azides. The 1, 3-dipolar cycloaddition is one of the simplest approaches for the construction of five-membered heterocyclic rings [7]. This method was discovered by Meldal and Sharpless [8, 9], which modified the conventional Huisgen 1, 3Dipolar Cycloaddition [10]. The chemical structures of synthesized compounds were confirmed by IR, ${ }^{1} \mathrm{H}$ NMR and ${ }^{13} \mathrm{C}$-NMR. These compounds were also screened for their in vitro antibacterial activities.

\section{MATERIAL AND METHODS:}

\subsection{Chemistry:}

All melting points are uncorrected and were determined by the Kofler bench. The ${ }^{1} \mathrm{H}-\mathrm{NMR}$ $(300 \mathrm{MHz})$ and ${ }^{13} \mathrm{C}-\mathrm{NMR}(75 \mathrm{MHz})$ spectra were recorded on Bruker using in $\mathrm{CDCl}_{3}$ as solvent and the TMS as internal standard, the chemical shifts are reported in ppm scale. The coupling constants (J) are expressed in hertz $(\mathrm{Hz})$ and singlet (s), doublet (d), doublet of a doublet (dd), and triplet (t) as well as multiplet (m). The analysis by X-ray was analyzed by Bruker APEX II CCD diffractometer Multi-scan SADABS2014/2. Compounds were routinely checked for their purity on Silica gel G (Merck), Iodine chamber and UV lamp were used for visualization of thin layer chromatography (TLC)[11].

\subsection{General procedure for the synthesis of the compounds 2.3.4:}

A mixture of compound 5-chloro- $1 \mathrm{H}$ indole-2,3-dione $(0,4 \mathrm{~g}, 2,20 \mathrm{mmol})$ in $\mathrm{N}$, Ndiméthylformamide $(15 \mathrm{ml})$ with $0,5 \mathrm{~g}(3,3 \mathrm{mmol})$ of $\mathrm{K}_{2} \mathrm{CO}_{3}$, BTBA $(0,1 \mathrm{~g}, 0,3 \mathrm{mmol})$ and 1.2 equiv of alkylating agent. The mixture is stirred for $48 \mathrm{~h}$ at room temperature. The precipitate was filtered and treated. The residue obtained was recrystallized from an ethanol solution in a yield. 


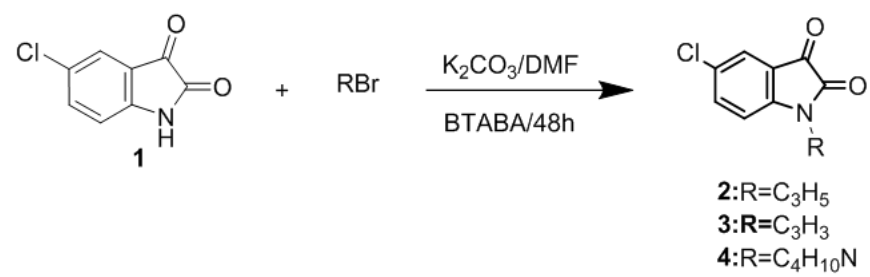

Figure1: Synthesis of the new 5-Chloroisatin derivatives

\subsection{Spectral data :}

Compound 2: 1-allyl-5-chloroindoline-2,3-dione: Yield: $89 \%$; m.p: $140-142^{\circ} \mathrm{C} ; \mathrm{R}_{\mathrm{f}}=0.78 .{ }^{1} \mathrm{H} \quad \mathrm{NMR}$ $\left(\mathrm{CDCl}_{3} ; 300 \mathrm{MHz}\right) \delta p p m$ 7.52-7.58 (m, 2H, $\left.\mathrm{H}_{\mathrm{Ar}}\right) ; 6.89$ $\left(\mathrm{d}, \mathrm{H}, \mathrm{H}_{\mathrm{Ar}},{ }^{3} \mathrm{~J}_{\mathrm{H}-\mathrm{H}}=9 \mathrm{~Hz}\right) ; 5.77-5.90(\mathrm{~m}, 1 \mathrm{H}, \mathrm{CH}) ; 5.30-$ $5.35\left(\mathrm{~m}, 2 \mathrm{H}, \mathrm{CH}_{2}\right) ; 4.38\left(\mathrm{~d}, 2 \mathrm{H}, \mathrm{CH}_{2},{ }^{4} \mathrm{~J}_{\mathrm{H}-\mathrm{H}}=3 \mathrm{~Hz}\right) .{ }^{13} \mathrm{C}$ NMR $\left(\mathrm{CDCl}_{3} ; 75 \mathrm{MHz}\right) \delta$ ppm: $182.18(\mathrm{C}=\mathrm{O}) ; 157.34$ $(\mathrm{N}-\mathrm{C}=\mathrm{O}) ; \quad 149.07, \quad 129.67,118.93(\mathrm{Cq}) ; 137.64$, $130.02, \quad 112.00 \quad\left(\mathrm{CH}_{\mathrm{Ar}}\right) ; 125.25 \quad(\mathrm{C}=\mathrm{CH}) ; 118.41$ $\left(\mathrm{C}=\mathrm{CH}_{2}\right) ; 42.63\left(\mathrm{CH}_{2}\right)$

Compound 3: 5-chloro-1-(prop-2-yn-1-yl)indoline2,3-dione: yield: $88 \%$; m.p: $166-170^{\circ} \mathrm{C} ; \mathrm{R}_{\mathrm{f}}=0.78 ;{ }^{1} \mathrm{H}$ NMR $\left(\mathrm{CDCl}_{3}\right) \delta$ ppm 7.57-7.62 (m, 2H, $\left.\mathrm{H}_{\mathrm{Ar}}\right) ; 7.12(\mathrm{~d}$, $\left.\mathrm{H}, \mathrm{H}_{\mathrm{Ar}},{ }^{3} \mathrm{~J}_{\mathrm{H}-\mathrm{H}}=6 \mathrm{~Hz}\right) ; 4.54\left(\mathrm{~s}, 2 \mathrm{H}, \mathrm{CH}_{2}\right) ; 2.34\left(\mathrm{t}, \mathrm{H},{ }^{4} \mathrm{~J}_{\mathrm{H}-}\right.$ $\mathrm{H}=3 \mathrm{~Hz}) ;{ }^{13} \mathrm{C} \mathrm{NMR}\left(\mathrm{CDCl}_{3}\right) \delta p p m: 181.55(\mathrm{C}=\mathrm{O})$; $156.60(\mathrm{~N}-\mathrm{C}=\mathrm{O}) ; 147.87,130.07,118.50(\mathrm{Cq}) ; 137.80$, 125.24, $112.75\left(\mathrm{CH}_{\mathrm{Ar}}\right) ; 73.72(\mathrm{C} \equiv \mathrm{C}) ; 71.21 \quad(\mathrm{CH})$; $29.59\left(\mathrm{CH}_{2}\right)$

Compound 4: 5-chloro-1-(2-(dimethylamino) ethyl) indoline-2,3-dione: Yield: $89 \%$; m.p : $114-116{ }^{\circ} \mathrm{C}$;
$\mathrm{R}_{\mathrm{f}}=0.78 ;{ }^{1} \mathrm{H}$ NMR $\left(\mathrm{CDCl}_{3} ; 300 \mathrm{MHz}\right) 7.53-7.54(\mathrm{~m}$, $\left.\mathrm{H}, \mathrm{H}_{\mathrm{Ar}}\right) ; 7.51\left(\mathrm{~d}, \mathrm{H}, \mathrm{H}_{\mathrm{Ar}},{ }^{3} \mathrm{~J}_{\mathrm{H}-\mathrm{H}}=9 \mathrm{~Hz}\right) ; 6.90\left(\mathrm{~d}, \mathrm{H}, \mathrm{H}_{\mathrm{Ar}}\right.$, $\left.{ }^{3} \mathrm{~J}_{\mathrm{H}-\mathrm{H}}=9 \mathrm{~Hz}\right) ; 3.85\left(\mathrm{t}, 2 \mathrm{H}, \mathrm{CH}_{2},{ }^{3} \mathrm{~J}_{\mathrm{H}-\mathrm{H}}=9 \mathrm{~Hz}\right) ; 3.75(\mathrm{t}$, $\left.2 \mathrm{H}, \mathrm{CH}_{2},{ }^{3} \mathrm{~J}_{\mathrm{H}-\mathrm{H}}=9 \mathrm{~Hz}\right) ; 2.15\left(\mathrm{~m}, 6 \mathrm{H}, \mathrm{CH}_{3}\right) ;{ }^{13} \mathrm{C} \mathrm{NMR}$ $\left(\mathrm{CDCl}_{3} ; 75 \mathrm{MHz}\right) \delta \mathrm{ppm}: 184.59(\mathrm{C}=\mathrm{O}) ; 164.45(\mathrm{~N}-$ $\mathrm{C}=\mathrm{O}) ; 146.22$, 141.13, $110.39(\mathrm{Cq}) ; 138.59,126.08$, $113.36\left(\mathrm{CH}_{\mathrm{Ar}}\right) ; 55.90,46.79\left(\mathrm{CH}_{2}\right) ; 45.09\left(\mathrm{CH}_{3}\right)$; Infra Red $v_{\max }(\mathrm{KBr}) / \mathrm{cm}^{-1}: 3565,3174,3081,2975,1720$, $1607,1445,1123,701,461$.

\subsection{General procedure for the synthesis of Cycloadduit:}

We add $0.2 \mathrm{~g}(0.903 \mathrm{mmol})$ of dipolarophile 2 , and 1.2 equiv of the nitrite oxide in $12 \mathrm{ml}$ of chloroform $\left(\mathrm{CHCl}_{3}\right)$, When the mixture reaches $0^{\circ} \mathrm{C}, 4$ $\mathrm{mL}$ of bleach $(\mathrm{NaOCl})$ are added dropwise, then the mixture is left stirring for 4 hours, the reaction is followed by TLC and the compound obtained purified and recrystallized from ethanol.

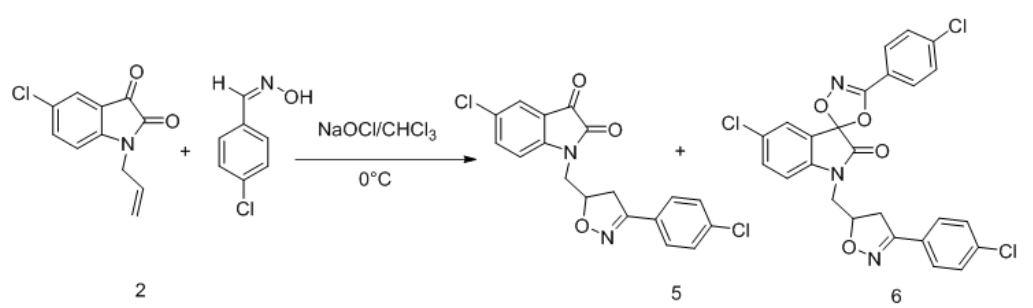

Figure2: Synthesis of the cycloadduits 5 and $\mathbf{6}$
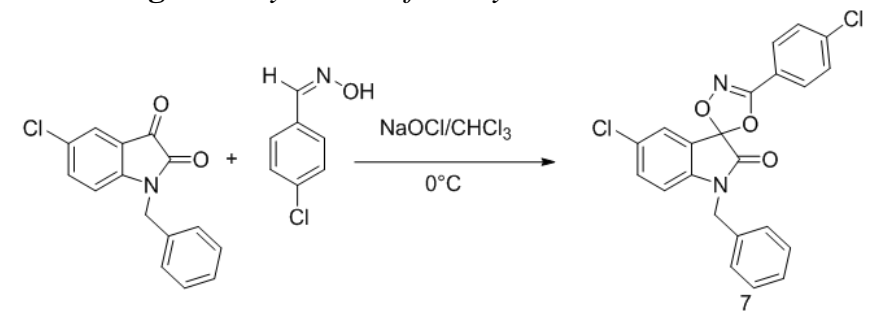

Figure3: synthesis of the new cycloadduit 7

\subsection{Spectral data :}

Compound 5: 5-chloro-1- ((3-(4-chlorophenyl) -4,5dihydroisoxazol-5-yl) methyl) indoline-2,3-dione : yield: $45 \%$; m.p : $210-215^{\circ} \mathrm{C} ; \mathrm{R}_{\mathrm{f}}=0.55 .{ }^{1} \mathrm{H}$ NMR $\left(\mathrm{CDCl}_{3} ; 300 \mathrm{MHz}\right) \delta \mathrm{ppm} 7.55-7.61\left(\mathrm{~m}, 4 \mathrm{H}, \mathrm{H}_{\mathrm{Ar}}\right) ; 7.40$ $\left(\mathrm{d}, 2 \mathrm{H}, \mathrm{H}_{\mathrm{Ar}},{ }^{3} \mathrm{~J}_{\mathrm{H}-\mathrm{H}}=9 \mathrm{~Hz}\right) ; 7.21\left(\mathrm{~d}, 1 \mathrm{H}, \mathrm{H}_{\mathrm{Ar}}{ }^{3} \mathrm{~J}_{\mathrm{H}-\mathrm{H}}=6 \mathrm{~Hz}\right)$; 5.04-5.14 (m, H, CH); 3.92-4.09 (qd, 2H, $\mathrm{CH}_{2},{ }^{3} \mathrm{~J}_{\mathrm{H}-\mathrm{H}}$ $\left.=18 \mathrm{~Hz}, \quad{ }^{4} \mathrm{~J}_{\mathrm{H}-\mathrm{H}}=6 \mathrm{~Hz},\right) ; 3.21-3.56\left(\mathrm{qd}, 2 \mathrm{H}, \mathrm{CH}_{2},{ }^{3} \mathrm{~J}_{\mathrm{H}-\mathrm{H}}\right.$ $\left.=12 \mathrm{~Hz}, \quad{ }^{4} \mathrm{~J}_{\mathrm{H}-\mathrm{H}}=6 \mathrm{~Hz}\right) ; . \quad{ }^{13} \mathrm{C} \quad \mathrm{NMR} \quad\left(\mathrm{CDCl}_{3}\right.$;

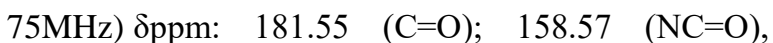
$149.45,145.81,136.69,130.03,127.12,116.76(\mathrm{Cq})$; $138.03,129.16,128.08,125.07,113.16\left(\mathrm{CH}_{\mathrm{Ar}}\right) ; 79.39$ $(\mathrm{CH}), 44.02,38.05\left(\mathrm{CH}_{2}\right)$

Compound 6: 5-chloro-3'- (4-chlorophenyl) -1- ((3(4-chlorophenyl) -4,5-dihydroisoxazol-5-yl) methyl) spiro [indoline-3,5'- [1,4,2] dioxazol]- 2-one: yield: $42 \%$; m.p : $224-228^{\circ} \mathrm{C} ; \mathrm{R}_{\mathrm{f}}=0.53{ }^{1} \mathrm{H}$ NMR $\left(\mathrm{CDCl}_{3}\right.$; $300 \mathrm{MHz})\left(\mathrm{CDCl}_{3}\right) \delta$ ppm $7.80\left(\mathrm{~d}, 2 \mathrm{H}, \mathrm{H}_{\mathrm{Ar}},{ }^{3} \mathrm{~J}_{\mathrm{H}-\mathrm{H}}=6 \mathrm{~Hz}\right)$; 
$7.50\left(\mathrm{~d}, 2 \mathrm{H}, \mathrm{H}_{\mathrm{Ar}},{ }^{4} \mathrm{~J}_{\mathrm{H}-\mathrm{H}}=3.6 \mathrm{~Hz}\right) ; 7.48-7.45(\mathrm{~m}, 6 \mathrm{H}$, $\left.\mathrm{H}_{\mathrm{Ar}}\right) ; 7.19\left(\mathrm{~d}, \mathrm{H}, \mathrm{H}_{\mathrm{Ar}},{ }^{3} \mathrm{~J}_{\mathrm{H}-\mathrm{H}}=9 \mathrm{~Hz},{ }^{4} \mathrm{~J}_{\mathrm{H}-\mathrm{H}}=6 \mathrm{~Hz}\right) ; 5.12-$ $5.05(\mathrm{~m}, \mathrm{H}, \mathrm{CH}) ; 3.87-4,06\left(\mathrm{qd}, \mathrm{H}, \mathrm{CH}_{2},{ }^{3} \mathrm{~J}_{\mathrm{H}-\mathrm{H}}=\right.$ $\left.15 \mathrm{~Hz},{ }^{4} \mathrm{~J}_{\mathrm{H}-\mathrm{H}}=6 \mathrm{~Hz}\right) ; 3.21-3.55\left(\mathrm{qd}, \quad \mathrm{H}, \quad \mathrm{CH}_{2},{ }^{3} \mathrm{~J}_{\mathrm{H}-\mathrm{H}}=\right.$ $\left.15 \mathrm{~Hz},{ }^{4} \mathrm{~J}_{\mathrm{H}-\mathrm{H}}=6 \mathrm{~Hz}\right) ; .{ }^{13} \mathrm{C} \mathrm{NMR}\left(\mathrm{CDCl}_{3} ; 75 \mathrm{MHz}\right) \delta \mathrm{ppm}$ : $168.12(\mathrm{NC}=\mathrm{O}) ; 166.03,162.00(\mathrm{C}=\mathrm{N}) ; 141.45$, $138.63,136.51,130.20,122.62,120.05(\mathrm{Cq}) ; 133.39$, $128.52, \quad 128.10, \quad 126.77, \quad 126.65, \quad 126.62,111.11$ $\left(\mathrm{CH}_{\mathrm{Ar}}\right) ; 79.39(\mathrm{CH}) ; 44.06,35.71\left(\mathrm{CH}_{2}\right)$.

Compound 7: 1-benzyl-5-chloro-3'- (4-chlorophenyl) spiro [indoline-3,5'- [1,4,2] dioxazol]-2-one: Yield: 54\%; m.p: $180-183 ;{ }^{1} \mathrm{H}$ NMR $\left(\mathrm{CDCl}_{3}\right) \delta$ ppm $7.80(\mathrm{~d}$, $\left.2 \mathrm{H}, \mathrm{H}_{\mathrm{Ar}},{ }^{3} \mathrm{~J}_{\mathrm{H}-\mathrm{H}}=8.6 \mathrm{~Hz}\right) ; 7.53\left(\mathrm{~d}, 2 \mathrm{H}, \mathrm{H}_{\mathrm{Ar}},{ }^{4} \mathrm{~J}_{\mathrm{H}-\mathrm{H}}\right.$ $=2.1 \mathrm{~Hz}) ; 7.48\left(\mathrm{~d}, 2 \mathrm{H}, \mathrm{H}_{\mathrm{Ar}},{ }^{3} \mathrm{~J}_{\mathrm{H}-\mathrm{H}}=8.7 \mathrm{~Hz}\right) ; 7.38-7.30(\mathrm{~m}$, $\left.5 \mathrm{H}, \mathrm{H}_{\mathrm{Ar}}\right) ; 6.72\left(\mathrm{~d}, \mathrm{H}, \mathrm{H}_{\mathrm{Ar}},{ }^{3} \mathrm{~J}_{\mathrm{H}-\mathrm{H}}=8.4 \mathrm{~Hz}\right) ; 1.71(\mathrm{~s}, 2 \mathrm{H}$,
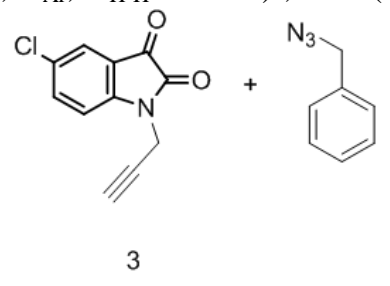

$\left.\mathrm{CH}_{2}\right) ;{ }^{13} \mathrm{C} \mathrm{NMR}\left(\mathrm{CDCl}_{3}\right) \delta$ ppm: $168.58(\mathrm{~N}-\mathrm{C}=\mathrm{O})$; $158.92(\mathrm{C}=\mathrm{N}) ; 142.42,138.47,134.13,129.49$, 122.87, $120.26(\mathrm{Cq}) ; 133.02,129.25,129.10,128.54$, $128.23,127.32,126.33,111.40\left(\mathrm{CH}_{\mathrm{Ar}}\right) ; 44.21\left(\mathrm{CH}_{2}\right)$.

\subsection{Synthesis of 1,2,3-triazoles by the Click Chemistry Method:}

$0.2 \mathrm{~g}$ of 5-chloro-1- (prop-2-ynyl) indoline2,3-dione and 1.2 equiv of benzyl azide are dissolved in $7 \mathrm{~mL}$ of ethanol at room temperature, 0.1 equiv of $\mathrm{CuSO}_{4} 5 \mathrm{H}_{2} \mathrm{O}$ and 0.2 equiv of sodium ascorbate dissolved in $7 \mathrm{~mL}$ of distilled water. The mixture is stirred for $24 \mathrm{~h}$, followed by TLC; the reaction crude is purified by chromatography on a silica gel column (ethyl acetate / Hexane (4/1)).

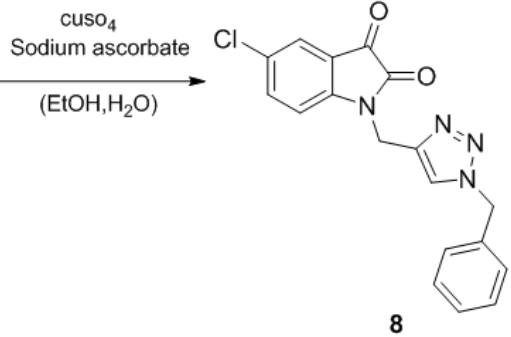

Figure4: Synthesis of 1-((1-benzyl-1H-1,2,3-triazol-4-yl)methyl)-5-chloroindoline-2,3-dione

\subsection{Spectral data :}

Compound 8: 1-((1-benzyl-1H-1,2,3-triazol-4-yl) methyl)- 5-chloroindoline-2,3-dione: Yield: 80\%; m.p : $\quad 140-145^{\circ} \mathrm{C} ; \quad \mathrm{R}_{\mathrm{f}}=0.55 ;{ }^{1} \mathrm{H} \quad \mathrm{NMR} \quad\left(\mathrm{CDCl}_{3}\right.$; $300 \mathrm{MHz}) \delta$ ppm 7.32-7.29 (m, 2H, $\left.\mathrm{H}_{\mathrm{Ar}}\right) ; 7.26(\mathrm{~d}, \mathrm{H}$, $\left.\mathrm{H}_{\mathrm{Ar}},{ }^{4} \mathrm{~J}_{\mathrm{H}-\mathrm{H}}=3 \mathrm{~Hz}\right) ; 7.08\left(\mathrm{~d}, 2 \mathrm{H}, \mathrm{H}_{\mathrm{Ar}},{ }^{4} \mathrm{~J}_{\mathrm{H}-\mathrm{H}}=3 \mathrm{~Hz}\right) ; 6.99$ $7.03(\mathrm{~m}, 1 \mathrm{H}, \mathrm{CH}) ; 6.71-6.73\left(\mathrm{~d}, 2 \mathrm{H}, \mathrm{H}_{\mathrm{Ar}},{ }^{4} \mathrm{~J}_{\mathrm{H}-\mathrm{H}}=3 \mathrm{~Hz}\right)$; 6.47-6.49 (m, H, H $\mathrm{Ar}_{\mathrm{Ar}}$ ); 5.23 (s, 2H, $\left.\mathrm{CH}_{2}\right) ; 4.89$ (s, 2H, $\left.\mathrm{CH}_{2}\right) ;{ }^{13} \mathrm{C} \mathrm{NMR}\left(\mathrm{CDCl}_{3} ; 75 \mathrm{MHz}\right) \delta \mathrm{ppm}: 186.50(\mathrm{C}=\mathrm{O})$; $165.30(\mathrm{~N}-\mathrm{C}=\mathrm{O}) ; 149.82,143.25,135.62,130.53$, $117.81(\mathrm{Cq})$; 132.65, 131.15, 129.47, 127.66, 123.11 $\left(\mathrm{CH}_{\mathrm{Ar}}\right) ; 125.65(\mathrm{CH}) ; 56.54,43.82\left(\mathrm{CH}_{2}\right)$.

\subsection{Antibacterial Activity}

The antibacterial activity of the synthesized compounds was tested against two bacteria Gram Pseudomonas aeruginosa, Escherichia coli, and two others Gram + : Bacillus cereus and Staphylococcus aureus using LB medium (Luria Bertani medium: yeast extract $5.0 \mathrm{~g}$, peptone $10.0 \mathrm{~g}$, sodium chloride $5.0 \mathrm{~g}$, distilled water $1000 \mathrm{~mL}$ ).

\subsection{A disc diffusion test}

As a first step, we have used a disc diffusion test us a primary antibacterial screening. This test was performed as recommended by CLSI [12]. Petri dishes containing LB agar medium were inoculated by bacterial suspension adjusted to a turbidity equivalent of an $0.5 \mathrm{McF}$ arland standard $\left(\approx 1.5 \times 10^{8} \mathrm{CFU} / \mathrm{mL}\right)$, and then, filter paper discs (about $6 \mathrm{~mm}$ in diameter) were deposited on top of the inoculated plates and impregnated with $5 \mu \mathrm{L}$ of test compounds. Test samples were dissolved in $2 \%$ of dimethyl sulfoxide (DMSO). The plates were incubated at $4^{\circ} \mathrm{C}$ for $2 \mathrm{~h}$ to permit good diffusion before incubation at $37 \pm 2{ }^{\circ} \mathrm{C}$ for $24 \mathrm{~h}$. Antibacterial activity of the extracts was evaluated by measuring the diameter of inhibition zone.

\subsection{Minimum inhibitory concentration determination (MIC):}

As a second step, we have proceeded to the determination of MICs of the products which have given positive results in the first test. The MIC was defined as the lowest concentration of compound giving complete inhibition of visible growth. All experiments were conducted in triplicates. MICs values were determined in 96 well-microplate using the micro-dilution assay according to the protocol described by Ismaili et al [13] with some modifications. Briefly, a stock solution of each product was prepared in $2 \%$ DMSO. Then, serial dilutions, of the antimicrobial agent were prepared in Mueller Hinton Broth (MHB) at final concentrations ranged between $5 \mathrm{mg} / \mathrm{mL}$ and $0.004 \mathrm{mg} / \mathrm{ml}$. Then, each well is inoculated with a microbial inoculum prepared in the same medium at a final concentration of $106 \mathrm{CFU} / \mathrm{mL}$. The 12 th tube was considered as growth control, because no extracts solutions were added. Then, $50 \mu \mathrm{L}$ of bacterial inoculum was added to each well at a final concentration of $106 \mathrm{CFU} / \mathrm{mL}$. The final concentration of the extracts was included from $5 \mathrm{mg} \mathrm{ml}-1$ (3rd well) to $0.019 \mathrm{mg} \mathrm{ml}^{-1}$ (11th 
well). After incubation at $37^{\circ} \mathrm{C}$ for $24 \mathrm{~h}, 10 \mu \mathrm{L}$ of rezasurin were added to each well as mycobacterial growth indicator. After further incubation at $37^{\circ} \mathrm{C}$ for 2 hours, the bacterial growth was revealed by reduction of blue dye resazurin to pink resorufin.

\subsection{Minimum bactericidal concentrations (MBC):}

As a third step, the minimum bactericidal concentrations (MBC) which is defined as the lowest concentration of antimicrobial agent needed to kill $99.9 \%$ of the final inoculum after incubation at $37^{\circ} \mathrm{C}$ for 24 hours was evaluated. The MBC can be determined after broth microdilution by spreading 5 $\mu \mathrm{L}$ from negative wells on Luria Bertani agar plates (Luria Bertani medium: yeast extract $5.0 \mathrm{~g}$, peptone $10.0 \mathrm{~g}$, sodium chloride $5.0 \mathrm{~g}$, distilled water $1000 \mathrm{ml}$ ).

\section{RESULTS AND DISCUSSION :}

The MIC and CMB values indicate that the synthesized 4 compound presented antimicrobial activity against all Gram-, Gram + bacteria tested, the 4 compound exhibited excellent inhibitory activity against Bacillus cereus at Gram positive with a MIC value of $0.156 \mathrm{mg} / \mathrm{ml}$. Whereas 2 and 3 had comparable activity against Staphylococcus aureus and Escherichia coli.

It should also be noted that the presence of a nitrogen atom in the case of the $\mathbf{4}$ compound increases the antimicrobial power. The presence of a nitrogen atom appears to be of great importance because it increases the antimicrobial potential.

\section{CONCLUSION}

To summarize, the series of novel 5Chloroisatin derivatives containing benzyl azide, chlorobenzaldehyde and the various brominated reagents were synthesized and characterized by ${ }^{1} \mathrm{H}$ NMR,${ }^{13} \mathrm{C}$ NMR. These derivatives were evaluated for their antibacterial activity in vitro against four bacteria including two gram positive and two gram negative. It is clear that the results revealed that the $\mathbf{4}$ compound was biologically active with different spectral activity across all bacteria studied, whereas compound $\mathbf{8}$ showed no antimicrobial effect. Whereas the other compounds 2, 3, 5, 6, 7 exhibited a moderate inhibitory effect.

\section{REFERENCES}

[1]. R Singh, A Chouhan. an overview of biological importance of pyrimidines. W.JPharma Pharmaceu.sc. 12(3)2014 574597.

[2]. SN. Pandeya, D Sriram, G Nath, E DeClercq, Synthesis, antibacterial, antifungal and antiHIV activities of Schiff and Mannich bases derived from isatin derivatives and N-[4-(4'-
Compound 7 exhibited a moderate inhibitory effect against Gram + and Gram- (Staphylococcus aureus and Escherichia coli) bacteria at a MIC value of $5 \mathrm{mg} / \mathrm{ml}$. However, the bacterium Pseudomonas aeruginosa was resistant to the $\mathbf{5}$ compound while compound $\mathbf{8}$ showed no antimicrobial effect against all strains tested. In general, gram-positive bacteria show areas of inhibition greater than those observed in Gram-negative bacteria in the case of the synthesized products.

Table1: MICs and MBCs of the compounds against the microbes used

\begin{tabular}{|c|c|c|c|c|}
\hline \multirow[t]{3}{*}{ compounds } & \multicolumn{4}{|c|}{$\operatorname{MCMBC}(\mathrm{mg} / \mathrm{m})$} \\
\hline & \multicolumn{2}{|c|}{ Gramt } & \multicolumn{2}{|c|}{ Gtam- } \\
\hline & Bucillus cereus & $\begin{array}{c}\text { Staphylococcus } \\
\text { aureus }\end{array}$ & Escherichiacoli & $\begin{array}{l}\text { Psendomonas } \\
\text { aeruginosa }\end{array}$ \\
\hline 2 & . & $0,1560,156$ & $0,02510,625$ & $\cdot$ \\
\hline 3 & - & $0,6250,0625$ & $2,5 / 2,5$ & - \\
\hline 4 & $0,15610,156$ & $0,313 / 0,313$ & $0,02510,625$ & $1,25 / 1,25$ \\
\hline 5 & - & - & - & $5 / 5$ \\
\hline 6 & - & - & - & - \\
\hline 7 & . & 5 & 5 & . \\
\hline 8 & - & - & - & \\
\hline
\end{tabular}

chlorophenyl)thiazol-2-yl] thiosemicarbazide official J. Eur Fed. Pharmaceut Sc. 9 (1) (1999) 25-31.

[3]. M. Swathi. K. Sarangapani. Synthesis and anti-inflammatory activity of a novel series of Isatin hydrazone and Isatin thio semicarbazone derivatives. World Journal of Pharmacy and Pharmaceutical sciences 2014;3(2):2070-8.

[4]. SA Khan, AA Siddiqui, S Bhatt. Analgesic activity of Isatin derivatives Asian. J. Chem, 14 (2002) 417-18.

[5]. VH Tran, QD Nguyen, NV Le. Study on the antituberculosis effect of some thiosemicarbazones and isonicotinyl hydrazone derivatives of Isatin and 5haloisatin. TapChiDou Hoc 8 (2000)15-7.

[6]. FD Popp, R Parson, BE Donigan. Synthesis of potential anticonvulsants: condensation of isatins with acetone and related ketones $\mathrm{J}$. pharmaceut. Sc, 69(10) (1980) 1235-7.

[7]. Z. Tribak, Y. Kandri Rodi, H. Elmsellem, I. Abdel-Rahman, A. Haoudi, M. K. Skalli, Y. Kadmi, B. Hammouti, M. Ali Shariati, E. M. Essassi, 5-chloro-1-octylindoline-2,3-dione as a new corrosion inhibitor for mild steel in hydrochloric acid solution. J. Mater. Environ. Sci. 8 (3) (2017) 1116-1127.

[8]. Z. Tribak, Y. Kandri Rodi, Y. Kharbach, A. Haoudi, M.K. Skalli, A. Mazzah, M. 
Akhazzane and E.M. Essassi synthesis of new 1H-indole-2,3-dione derivatives using phasetransfer catalysis and characterization by $\mathrm{x}$ ray crystallography J.Mar. Chim. Heterocycl 15(1) (2016)79-84.

[9]. V. V Rostovtsev., L.G., Green V. V Fokin., K.B Sharpless., A Stepwise Huisgen Cycloaddition Process: Copper(I)-Catalyzed Regioselective "Ligation" of Azides and Terminal Alkynes Angew. Chem. Int. Ed. 41 (2002) 2596-2599.

[10]. Z. Tribak, Y. Kandri Rodi, A. Haoudi, M.K. Skalli, A. Mazzah, M. Akhazzane, E.M. Essassi. cycloaddition 1,3-dipolaire des derives de la 5-chloro-1H-indole-2,3 dione: vers de nouvelles isoxazolines et spirodioxazolines. J.Mar. Chim. Heterocycl 16(1)(2016)58-65

[11]. Z. Tribak, A. Haoudi. Y. Kandri Rodi. H Elmsellem, M.K. Skalli. Y. Ouzidan, A. Mazzah, E.M. Essassi. Synthesis and reactivity of new heterocyclic systems derived from 5-chloro-1H-indole-2,3-dione.Mor. J. Chem. 4(4) (2016)1157-1163

[12]. CLSI, Performance Standards for Antimicrobial Disk Susceptibility Tests, Approved Standard, 7th ed., CLSI document M02-A11. Clinical and Laboratory Standards Institute, 950 West Valley Road, Suite 2500, Wayne, Pennsylvania 19087, USA, 2012.

[13]. Ismaili, L. Milella, S. Fkih-Tetouani, A. Ilidrissi, A. Camporese, S. Sosa, G. Altinier, R.D Loggia, R Aquino. In vivo topical antiinflammatory and in vitro antioxidant activities of two extracts of Thymus satureioides leaves 91 (2004) 31- 